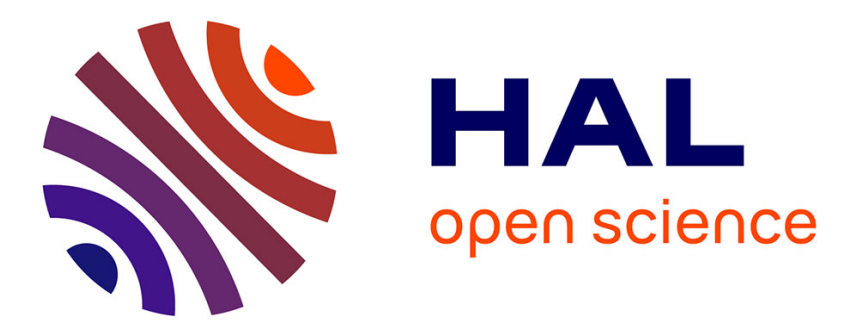

\title{
Dispersion relation analysis of the neutral kaon regeneration amplitude in carbon
}

\author{
A. Angelopoulos, A. Apostolakis, G. Backenstoss, E. Aslanides, P. Bargassa, \\ O. Behnke, A. Benelli, V. Bertin, F. Blanc, P. Bloch, et al.
}

\section{- To cite this version:}

A. Angelopoulos, A. Apostolakis, G. Backenstoss, E. Aslanides, P. Bargassa, et al.. Dispersion relation analysis of the neutral kaon regeneration amplitude in carbon. European Physical Journal C: Particles and Fields, 1999, 10, pp.19-25. in2p3-00012094

\section{HAL Id: in2p3-00012094 https://hal.in2p3.fr/in2p3-00012094}

Submitted on 29 Mar 1999

HAL is a multi-disciplinary open access archive for the deposit and dissemination of scientific research documents, whether they are published or not. The documents may come from teaching and research institutions in France or abroad, or from public or private research centers.
L'archive ouverte pluridisciplinaire HAL, est destinée au dépôt et à la diffusion de documents scientifiques de niveau recherche, publiés ou non, émanant des établissements d'enseignement et de recherche français ou étrangers, des laboratoires publics ou privés. 


\title{
EUROPEAN ORGANIZATION FOR NUCLEAR RESEARCH
}

CERN-EP/99-34

February 26, 1999

\section{Dispersion relation analysis of the neutral kaon regeneration amplitude in carbon}

\section{CPLEAR Collaboration}

A. Angelopoulos ${ }^{1)}$, A. Apostolakis ${ }^{1)}$, E. Aslanides ${ }^{11)}$, G. Backenstoss ${ }^{2)}$, P. Bargassa $^{13)}$, O. Behnke ${ }^{17)}$, A. Benelli ${ }^{2)}$, V. Bertin ${ }^{11)}$, F. Blanc ${ }^{7,13)}$, P. Bloch ${ }^{4)}$, P. Carlson ${ }^{15)}$, M. Carroll ${ }^{9)}$, E. Cawley ${ }^{9)}$, M.B. Chertok ${ }^{3)}$, M. Danielsson ${ }^{15)}$, M. Dejardin ${ }^{14)}$, J. Derre ${ }^{14)}$, A. Ealet ${ }^{11)}$, C. Eleftheriadis ${ }^{16)}$, L. Faravel ${ }^{7)}$, W. Fetscher ${ }^{17)}$, M. Fidecaro ${ }^{4)}$, A. Filipčič ${ }^{10)}$, D. Francis ${ }^{3)}$, J. Fry ${ }^{9)}$, E. Gabathuler ${ }^{9)}$, R. Gamet ${ }^{9)}$, H.-J. Gerber ${ }^{17)}$, A. Go ${ }^{4)}$, A. Haselden ${ }^{9}$, P.J. Hayman ${ }^{9)}$, F. Henry-Couannier ${ }^{11)}$, R.W. Hollander ${ }^{6)}$, K. Jon-And ${ }^{15)}$, P.-R. Kettle ${ }^{13)}$, P. Kokkas ${ }^{4)}$, R. Kreuger ${ }^{6)}$, R. Le Gac ${ }^{11)}$, F. Leimgruber ${ }^{2)}$, I. Mandić( ${ }^{10)}$, N. Manthos ${ }^{8)}$, G. Marel ${ }^{14)}$, M. Mikuž ${ }^{10)}$, J. Miller ${ }^{3)}$, F. Montanet ${ }^{11)}$, A. Muller ${ }^{14)}$, T. Nakada ${ }^{13)}$, B. Pagels ${ }^{17)}$, I. Papadopoulos ${ }^{16)}$, P. Pavlopoulos ${ }^{2)}$, G. Polivka ${ }^{2)}$, R. Rickenbach ${ }^{2)}$, B.L. Roberts ${ }^{3)}$, T. Ruf ${ }^{4}$, M. Schäfer ${ }^{17)}$, L.A. Schaller ${ }^{7)}$, T. Schietinger ${ }^{2)}$, A. Schopper ${ }^{4)}$, L. Tauscher ${ }^{2)}$, C. Thibault $^{12)}$, F. Touchard ${ }^{11)}$, C. Touramanis ${ }^{9)}$, C.W.E. Van Eijk $^{6)}$, S. Vlachos ${ }^{2)}$, P. Weber ${ }^{17)}$, O. Wigger ${ }^{13)}$, M. Wolter ${ }^{17)}$, D. Zavrtanik ${ }^{10)}$, D. Zimmerman ${ }^{3)}$

and

M.P. Locher ${ }^{18)}$, V.E. Markushin ${ }^{18)}$

\begin{abstract}
We apply a forward dispersion relation to the regeneration amplitude for kaon scattering on ${ }^{12} \mathrm{C}$ using all available data. The CPLEAR data at low energies allow the determination of the net contribution from the subthreshold region which turns out to be much smaller than earlier evaluations, solving a long standing puzzle.
\end{abstract}

to be submitted to The European Physical Journal C

\footnotetext{
1) University of Athens, Greece

2) University of Basle, Switzerland

3) Boston University, USA

4) CERN, Geneva, Switzerland

5) LIP and University of Coimbra, Portugal

6) Delft University of Technology, Netherlands

7) University of Fribourg, Switzerland

8) University of Ioannina, Greece

9) University of Liverpool, UK

10) J. Stefan Inst. and Phys. Dep., University of Ljubljana, Slovenia

11) CPPM, IN2P3-CNRS et Université d'Aix-Marseille II, France

12) CSNSM, IN2P3-CNRS, Orsay, France

13) Paul Scherrer Institut (PSI), Villigen, Switzerland

14) CEA, DSM/DAPNIA, CE-Saclay, France

15) Royal Institute of Technology, Stockholm, Sweden

16) University of Thessaloniki, Greece

17) ETH-IPP Zürich, Switzerland

18) Paul Scherrer Institut (PSI), Theory Group, Villigen, Switzerland
} 
Introduction

Regeneration of neutral kaons is an excellent tool to study kaon-nuclear scattering [1] and of crucial importance to experiments measuring $\mathrm{CP}$ violation in the kaon system [2]. In the latter context, CPLEAR [3] has determined the kaon regeneration amplitude on $\mathrm{C}$ in the energy range between 0.56 and $0.9 \mathrm{GeV}$. In the present paper we explore the consequence of these data for strong-interaction physics analyzing the kaon-carbon scattering amplitude in the classical framework of forward dispersion relations (FDR) using in addition all the available information on KC scattering ${ }^{1)}$ : the kaon regeneration on carbon [4-14], total cross sections [15-18], near forward differential cross sections [17], and the scattering length from kaonic atoms [19,20]. For high energies, we use the Regge model which is well supported experimentally [13,14]. For the low energy application presented here this last aspect is not important. The energy range of the CPLEAR experiment is however well suited to obtain information on the strong-interaction physics occurring below the elastic threshold, often termed the unphysical region. Our results indicate that previous evaluations of FDRs have to be revised entirely in this region. Due to the new data situation, the net contribution from the unphysical region turns out to be much smaller and a simple coherent sum of elementary $\mathrm{KN}$ amplitudes is inadequate and even has the wrong sign as will be discussed below.

\section{Selection and treatment of data}

We define the regeneration amplitude as the difference between the nuclear amplitudes of forward scattering of $\overline{\mathrm{K}^{0}}$ and $\mathrm{K}^{0}$ on $\mathrm{C}$ :

$$
f^{(-)}(\omega)=f\left(\overline{\mathrm{K}^{0} \mathrm{C}} ; \omega, \theta=0\right)-f\left(\mathrm{~K}^{0} \mathrm{C} ; \omega, \theta=0\right)
$$

where $\omega$ is the total laboratory energy of the kaon. Note that this definition, which we choose for compatibility with earlier evaluations of FDR (an alternative definition with an extra factor $1 / 2$ in the r.h.s. of Eq. 1 is also used in the literature), has the opposite sign when compared to the $\Delta f$ of our earlier regeneration analysis [3].

Table 1 summarizes all experimental efforts to determine the regeneration amplitude in carbon. Most regeneration experiments determined modulus and phase of $f^{(-)}$from $\pi^{+} \pi^{-}$decay rates behind a regenerator [3-5,7,9-14]. Two groups $[6,8]$ also obtained a phase measurement from the time-dependent charge-asymmetry in semileptonic decays, thus independent of the $\mathrm{CP}$ violation phase $\phi_{+-}$.

Owing to the isospin symmetry of the ${ }^{12} \mathrm{C}$ nucleus, $\mathrm{K}^{ \pm}$data may be used to extract information on the regeneration amplitude, if charge invariance is assumed:

$$
f^{(-)}(\omega)=f\left(\mathrm{~K}^{-} \mathrm{C} ; \omega, \theta=0\right)-f\left(\mathrm{~K}^{+} \mathrm{C} ; \omega, \theta=0\right)
$$

Then, with the use of the optical theorem, the difference between the total cross sections of $\mathrm{K}^{-}$ and $\mathrm{K}^{+}$is a measure of the imaginary part of $f^{(-)}$[15-18] (Table 2). One of these experiments [17] also derived values for the real part from a measurement of the differential cross section $\mathrm{d} \sigma / \mathrm{d} q^{2}$.

Similarly, the $\mathrm{K}^{-} \mathrm{C}$ scattering length, $a_{0}\left(\mathrm{~K}^{-} \mathrm{C}\right)$, extracted [20] from X-ray transition data obtained with kaonic carbon [19] may be regarded as a measurement of the $\overline{\mathrm{K}}^{0}$ scattering amplitude near zero kinetic energy. The corresponding amplitude for $\mathrm{K}^{0}$, having no inelastic channels open below threshold, must be purely real such that $\operatorname{Im} f^{(-)}\left(m_{\mathrm{K}^{0}}\right)=-\operatorname{Im} a_{0}\left(\mathrm{~K}^{-} \mathrm{C}\right)$.

Where appropriate, the results have been adapted to the current world averages for the neutral kaon parameters, such as $\Delta m, \tau_{\mathrm{S}}$ and $\eta_{+-}$, taken from Ref. [21]. Here it is important to

1) All the data refer to natural carbon, with a $98.9 \%$ of ${ }^{12} \mathrm{C}$ content, to which the analysis applies. 
note that some of these averages, in particular $\phi_{+-}$, are themselves influenced by regeneration measurements so that care must be taken when employing them to correct these very measurements. Such feedback effects, however, were found to be very small and may be neglected in our analysis.

The corrected data are illustrated in Figs. 1-4, separately for measurements of modulus, phase, imaginary and real part of $f^{(-)}$. Fig. 3 reveals a conspicuous systematic disagreement between the total cross section results of Ref. [17] and the data of the other three experiments at the same energies. We exclude the data published in Ref. [17] from our fit, imaginary parts as well as real parts, as the latter directly depend on the former. For their impact see Sec. 4.

\section{The dispersion relation and its parameterization}

In the framework of local relativistic field theories, analyticity of the amplitude has been proven to follow from local causality and it is therefore very natural to assume it as well for kaon scattering off nuclei. We use an unsubtracted dispersion relation as it is readily derived for antisymmetric amplitudes [22]. Since the precision of the data does not allow fitting several pole terms, they are represented by a single effective pole:

$$
\operatorname{Re} f^{(-)}(\omega)=\frac{2 \omega r}{\omega^{2}-\omega_{\mathrm{p}}^{2}}+\frac{2 \omega}{\pi} \mathcal{P} \int_{\omega_{\Lambda}}^{\infty} \mathrm{d} \omega^{\prime} \frac{\operatorname{Im} f^{(-)}\left(\omega^{\prime}\right)}{\omega^{\prime 2}-\omega^{2}}
$$

where $\omega$ denotes the total laboratory energy of the kaon and $\mathcal{P}$ stands for principal value. The effective pole term contains two parameters, position $\omega_{\mathrm{p}}$ and residue $r$. The threshold $\omega_{\Lambda}$ marks the beginning of the unphysical cut, i.e., the energy at which production of a free $\Lambda$ becomes possible, $\omega_{\Lambda}=183.6 \mathrm{MeV}$. The integration over the imaginary part starts at $\omega_{\Lambda}$ and extends all the way to infinity. To carry out the integration, a parameterization of $\operatorname{Im} f^{(-)}$is needed over this range. Guided by the data and theoretical expectations, we have chosen the following parameterization:

- The Regge model with one pole trajectory exchange [23] is applied at energies above a certain energy $\omega_{\mathrm{k}}$, giving

$$
\operatorname{Im} f^{(-)}(\omega)=\beta\left(\omega / \omega_{0}\right)^{\alpha} \sin \left(\frac{\pi}{2}[\alpha+1]\right) .
$$

We choose the dimensional parameter $\omega_{0}=1 \mathrm{MeV}$.

- In the intermediate energy range around $1 \mathrm{GeV}$, the total cross section data clearly indicate a resonance. We parameterize it for simplicity as a Gaussian on a background, the shape of which we describe by the same simple power law as employed at high energies, but with a different exponent:

$$
\operatorname{Im} f^{(-)}(\omega)=b\left(\omega / \omega_{0}\right)^{a} \sin \left(\frac{\pi}{2}[a+1]\right)+C \exp \left(-\frac{1}{2}\left[\frac{\omega-\omega_{\text {res }}}{\sigma}\right]^{2}\right)
$$

The transition energy $\omega_{\mathrm{k}}$ follows from $\alpha, \beta, a$ and $b$ if the function $\operatorname{Im} f^{(-)}(\omega)$ is to be continuous. In the fit, we will vary $\omega_{\mathrm{k}}$ in place of $b$ as a free parameter.

- The sensitivity of the CPLEAR data to the imaginary part of the regeneration amplitude is not sufficient for a parameter fit in the energy range below the first total cross section measurement at $847 \mathrm{MeV}$. Following Refs. [24] and [25], we simply assume an essentially linear increase of $\operatorname{Im} f^{(-)}$from the physical threshold to $830 \mathrm{MeV}$ total laboratory energy, in accordance with the data. 
- At the physical threshold, we fix $\operatorname{Im} f^{(-)}$to the value corresponding to the measured $\mathrm{K}^{-} \mathrm{C}$ scattering length. The small error on this measurement is negligible in this analysis.

- Virtually nothing is known about $\operatorname{Im} f^{(-)}(\omega)=\operatorname{Im} f\left(\overline{\mathrm{K}}^{0} \mathrm{C} ; \omega, \theta=0\right)$ below the elastic threshold, except that it must vanish at the beginning of the unphysical cut $\omega_{\Lambda}$. We find that any smooth and small contribution from the integral between $\omega_{\Lambda}$ and $m_{\mathrm{K}^{0}}$ in (3) is compatible with the experimental information, so that we may assume a linear behavior for this energy range as well.

This gives a total of nine parameters to be determined in the fit. All of these parameters result from the interpolation of the data except for the effective pole, which represents the unphysical region below the elastic threshold. It turns out, however, that our evaluations are quite insensitive to the pole position $\omega_{\mathrm{p}}$. We therefore fix it to the position of the $\Lambda$ pole, given by the total energy needed to form a ${ }^{12} \mathrm{C}_{\Lambda}$ resonance,

$$
s=m_{\mathrm{C}_{\Lambda}}^{2}=m_{\mathrm{K}^{0}}^{2}+m_{\mathrm{C}}^{2}+2 \omega_{\mathrm{p}} m_{\mathrm{C}}
$$

which gives $\omega_{\mathrm{p}}=172.3 \mathrm{MeV}$.

Further parameters are needed for experimental reasons. As is evident from Fig. 1, there are systematic shifts in the normalization of the moduli measured by the high energy experiments (parameter $\beta$ ) whereas there is good agreement on the slope parameter $\alpha$. To prevent the systematic normalization uncertainties from affecting the result on $\alpha$, we allow for three additional correction factors, $N_{\mathrm{Car}}, N_{\mathrm{Alb}}$ and $N_{\text {Roe }}$ for the Brookhaven [10], Serpukhov [11,12] and Fermilab [13] experiments, respectively, to be determined together with the other parameters in the fit. Since the later Fermilab publication [14] does not contain individual data points but only the value of $\alpha$ resulting from their analysis, we directly employ this value in our fit.

\section{$4 \quad$ Fit results and discussion}

We now vary the 11 parameters defined in the preceding section and compare at each data point the prediction for the real part from (3) with the experiment. Correlations between different measured quantities are taken into account where they have been reported, in particular the strong correlations between our own measurements of imaginary and real parts [3]. Uncertainties in the kaon beam energy are neglected throughout. The weighted deviations are then summed up to a $\chi^{2}$ in the usual way. Minimization of this $\chi^{2}$ therefore reflects both the measured imaginary and real parts of the amplitude and yields the following parameters $\left(\chi^{2}=134.2\right.$ for 127 degrees of freedom):

$$
\begin{array}{rc}
r= & -1.57 \pm 0.25 \\
C & =(2.55 \pm 0.18) \mathrm{fm} \\
\omega_{\text {res }} & =(1130 \pm 9) \mathrm{MeV} \\
\sigma & =(147 \pm 12) \mathrm{MeV} \\
\omega_{\mathrm{k}} & =\left(4570_{-330}^{+360}\right) \mathrm{MeV} \\
a & =0.61 \pm 0.03 \\
\alpha & =0.424 \pm 0.005 \\
\beta & =(0.35 \pm 0.02) \mathrm{fm} \\
N_{\text {Car }}= & 0.96 \pm 0.02 \\
N_{\text {Alb }}= & 1.22 \pm 0.07 \\
N_{\text {Roe }}= & 1.07 \pm 0.03
\end{array}
$$


The resulting fit functions are shown in Figs. 1-4, together with an optical model calculation [26].

In order to discuss the physics below the elastic threshold we introduce the discrepancy function [22]

$$
\Delta(\omega)=\operatorname{Re} f_{\exp }^{(-)}(\omega)-\operatorname{Re} f_{\text {phys }}^{(-)}(\omega)
$$

where Re $f_{\text {phys }}^{(-)}(\omega)$ represents the dispersion integral (Eq. 3) from the physical region $\left.{ }^{2}\right)(\omega>$ $m_{\mathrm{K}^{0}}$ ). If the unphysical region is well represented in the physical region by the effective pole assumed in Eq. 3 then the quantity

$$
D(\omega)=\frac{\omega^{2}-\omega_{\mathrm{p}}^{2}}{2 \omega} \Delta(\omega)
$$

should be independent of the energy and equal to the effective residue ${ }^{3)}$,

$$
D(\omega)=r .
$$

In Fig. 5 we show $D(\omega)$ for $\omega_{\text {p }}$ from Eq. 6 corresponding to a ${ }^{12} \mathrm{C}_{\Lambda}$ system. The function $D(\omega)$ is not sensitive to the exact pole position and Fig. 5 shows no energy dependence which confirms the validity of the effective pole ansatz. The effective residue turns out to be small and negative while previous attempts to determine it from FDR led to large and positive values:

$$
r=\left\{\begin{array}{rll}
-1.57 \pm 0.25 & \text { a) } & \text { this analysis } \\
23.2 \pm 5.8 & \text { b) } & \text { Ref. [24] } \\
12.2 \pm 3.0 & \text { c) } & \text { Ref. [25] }
\end{array}\right.
$$

The main reason for this is that both of these earlier analyses completely relied on the data of Ref. [17] which we have discarded in view of their incompatibility with all other experiments. Given the large error bars of these data, their inclusion into our fit hardly changes the pole residue but increases the $\chi^{2}$ to 197.6 for now 133 degrees of freedom. Without the CPLEAR data for the real part, we would get a large positive residue of order of $r=12$, as can be seen from Fig. 5, consistent with the earlier analysis [25].

Our main result therefore is that the residue is small. In our analysis even its negativity is stable against considerable variations of a smooth background in the unphysical region and against variations of the imaginary part in the physical region below $830 \mathrm{MeV}$. However, since the contribution of the effective pole is not large where the real parts are measured by CPLEAR, $0.56 \leq \omega \leq 0.9 \mathrm{GeV}$, the sign is less certain than the magnitude.

From first principles the sign of the residue is directly related to the parity of the system of the particles exchanged [22]. For the elementary $\mathrm{K}^{ \pm} \mathrm{p}$ scattering, hyperon exchange leads to a positive residue (similar to $\pi \mathrm{N}$ scattering). Hence if the unphysical region for $\mathrm{K}^{12} \mathrm{C}$ scattering were dominated by the superposition of elementary hyperon exchange of Fig. 6a, a positive residue would be expected. If, however, an additional pion in a relative $\mathrm{S}$-wave is exchanged (Fig. 6b) or if the hyperon in Fig. 6a is in P-wave relative to the spectator nucleons, then the opposite parity results and the residue is negative. Our analysis clearly indicates that there are large cancellations between these contributions with different parities since the residue is stably small. The smallness of the effective residue is actually very gratifying since the earlier results

\footnotetext{
2) To avoid a singularity at $\omega=m_{\mathrm{K}^{0}}$ in $f_{\text {phys }}^{(-)}(\omega)$ we use a linear parameterization of $\operatorname{Im} f^{(-)}(\omega)$ for $\omega<m_{\mathrm{K}^{0}}$ as described in Section 3. Its contribution to $\Delta(\omega)$ is negligible in the energy range of CPLEAR data.

3) More elaborated methods (see [28]) do not help in our case because of the scarcity of data.
} 
$[24,25]$ claiming a large and positive residue are very hard to understand. As was already noticed by Dumbrajs [25], the impulse approximation has no chance to work. He observed that the measured imaginary part (total cross section) at low energies are much smaller than the impulse approximation while the result for the effective residue (10c) is about $50 \%$ larger than the sum of the elementary residues for $\Lambda$ and $\Sigma$ exchanges. The value (10b) from [24] is even bigger. Our result (10a) therefore solves a long standing puzzle.

The threshold region deserves some comment. In view of the large and repulsive $\mathrm{K}^{-} \mathrm{C}$ scattering length [20] we have also used the near threshold parameterization of [24] where this feature is related to a narrow peak in the imaginary part below threshold, see Fig. 3. This local structure near threshold does not affect our parameters for the effective pole, as we have checked. Our evaluation gives for the real part of the regeneration amplitude at threshold $\operatorname{Re} f^{(-)}\left(m_{\mathrm{K}^{0}}\right)=2.9 \mathrm{fm}$. Using the real part of the $\mathrm{K}^{-} \mathrm{C}$ scattering length $\operatorname{Re} a_{0}\left(\mathrm{~K}^{-} \mathrm{C}\right)=$ $2.9 \mathrm{fm}$ from Ref. [20] we obtain the real part of the $\mathrm{K}^{+} \mathrm{C}$ scattering length $\operatorname{Re} a_{0}\left(\mathrm{~K}^{+} \mathrm{C}\right)=$ $\operatorname{Re} f^{(-)}\left(m_{\mathrm{K}^{0}}\right)+\operatorname{Re} a_{0}\left(\mathrm{~K}^{-} \mathrm{C}\right)=5.8 \mathrm{fm}$. The value of $a_{0}\left(\mathrm{~K}^{+} \mathrm{C}\right)$ should be considered as an estimate: it is sensitive to the parameterization of $\operatorname{Im} f^{(-)}(\omega)$ near the elastic threshold because of a steep energy dependence of $\operatorname{Re} f^{(-)}(\omega)$ (see Fig.4). For comparison, using an optical model, we obtain $a_{0}\left(\mathrm{~K}^{+} \mathrm{C}\right) \approx 1.5 \mathrm{fm}$. Obviously there is a partial cancellation between the contributions from $\mathrm{K}^{-}$and $\mathrm{K}^{+}$for $f^{(-)}$since both scattering lengths are repulsive. While these findings are interesting for the discussion of scattering lengths and the detailed behavior of the amplitudes near threshold, these local structures are irrelevant for our discussion of the effective pole which is dominated by much larger energy scales (the energy range of the CPLEAR experiment).

In summary, we conclude that the combined information on the $\mathrm{K}^{12} \mathrm{C}$ and $\overline{\mathrm{K}}^{12} \mathrm{C}$ amplitudes is very well described by a forward dispersion relation containing only a small effective pole contribution from the subthreshold region, consistent with theoretical expectations. Earlier evaluations claiming a large contribution from the unphysical region can be excluded on the basis of the measured regeneration amplitude from CPLEAR in combination with the information on this reaction from all other consistent experimental data. These results are of relevance in the analysis of nuclear scattering and of CP-violation experiments at low energy [29].

\section{Acknowledgements}

We thank J. Ellis and N.E. Mavromatos for helpful discussions in the earlier stages of this analysis. This work was supported by the following institutions: the French CNRS/Institut de Physique Nucléaire et de Physique des Particules, the French Commissariat à l'Energie Atomique, the Greek General Secreteriat of Research and Technology, the Netherlands Foundation for Fundamental Research on Matter (FOM), the Portuguese JNICT, the Ministry of Science and Technology of the Republic of Slovenia, the Swedish Natural Science Research Council, the Swiss National Science Foundation, the UK Particle Physics and Astronomy Research Council (PPARC), and the US National Science Foundation.

\section{References}

[1] A. Gsponer et al., Phys. Rev. Lett. 42 (1979) 13;

V.L. Telegdi, $\mathrm{K}_{\mathrm{S}}$-regeneration: an interplay of nuclear and particle physics, in: AIP Conf. Proc. No. 26 (International Conference on High-Energy Physics and Nuclear Structure, Santa Fe and Los Alamos, June 1975), eds. D.E. Nagle et al. (American Institute of Physics, New York, 1975) p. 289.

[2] See for instance contributions to round-table discussion on regeneration phase measurement by A. Martin, K. Kleinknecht and B. Winstein, in: Proc. of the Workshop on K 
Physics (Orsay, 1996), ed. L. Iconomidou-Fayard (Editions Frontières, Gif-sur-Yvette Cedex, 1997) p. 329-342.

[3] A. Angelopoulos et al., CPLEAR Collaboration, Phys. Lett. B 413 (1997) 422.

[4] J.H. Christenson, J.W. Cronin, V.L. Fitch, and R. Turlay, Phys. Rev. 140 (1965) B74.

[5] A. Böhm et al. (1966) unpublished, cited in K. Kleinknecht, Fortschritte der Physik 21 (1973) 57 (p. 71); K. Kleinknecht, private communication.

[6] M. Bott-Bodenhausen et al., Phys. Lett. 24B (1967) 438.

[7] M. Bott-Bodenhausen et al., Interference between $K_{L}$ and $K_{S}$ amplitudes in the $\pi^{+} \pi^{-}$ decay mode, in: Proc. Topical Conference on Weak Interactions (Geneva, January 1969), CERN 69-7 (Geneva, 1969) p. 329.

[8] W.C. Carithers et al., Phys. Rev. Lett. 34 (1975) 1240.

[9] W.C. Carithers et al., Phys. Rev. Lett. 34 (1975) 1244.

[10] W.C. Carithers et al., Nucl. Phys. B 118 (1977) 333.

[11] J. Hladký et al., Czech. J. Phys. B 26 (1976) 1290.

[12] K.-F. Albrecht et al., Nucl. Phys. B 93 (1975) 237.

[13] J. Roehrig et al., Phys. Rev. Lett. 38 (1977) 1116, ibid 39 (1977) 674 (E).

[14] B. Schwingenheuer et al., Phys. Rev. Lett. 74 (1995) 4376.

[15] D.V. Bugg et al., Phys. Rev. 168 (1968) 1466.

[16] R.J. Abrams et al., Phys. Rev. D 4 (1971) 3235.

[17] B. Gobbi, W. Hakel, J.L. Rosen, and S. Shapiro, Phys. Rev. Lett. 29 (1972) 1278, 1281.

[18] V.N. Afonasyev et al., Yad. Phys. 47 (1988) 1656.

[19] G. Backenstoss et al., Phys. Lett. 38B (1972) 181.

[20] R. Seki, Phys. Rev. Lett. 29 (1972) 240.

[21] Particle Data Group, R.M. Barnett et al., Phys. Rev. D 54 (1996) 1.

[22] T.E.O. Ericson and M.P. Locher, Nucl. Phys. A 148 (1970) 1.

[23] N. Cabibbo, Phys. Lett. 22 (1966) 212.

[24] K. Arai, I. Endo and M. Kikugawa, Prog. Theor. Phys. 56 (1976) 1345.

[25] O. Dumbrajs, Nuovo Cimento 54 A (1979) 155, see also: Kaon-nucleus scattering, regeneration and analyticity, in: Proc. Low and Intermediate Energy Kaon-nucleon Physics (Rome, March 1980), eds. E. Ferrari and G. Violini (D. Reidel, Holland, 1981) p. 187.

[26] P.H. Eberhard, F. Uchiyama, Nucl. Instr. and Meth. A 350 (1994) 144.

[27] R. Briere, Ph.D. thesis, University of Chicago, 1995 (unpublished), and private communication.

[28] J. Antolín, Phys. Rev. D 43 (1991) 1532, J. Math. Phys. 31 (1990) 791;

J. Antolín and A. Cruz, J. Math. Phys. 27 (1986) 104, J. Phys. G: Nucl. Phys. 12 (1986) 297.

[29] L. Maiani et al. (eds.), The Second DA $\Phi N E$ Physics Handbook (INFN, Frascati, 1995). 
Table 1: Carbon regeneration experiments.

\begin{tabular}{|c|c|c|c|c|c|}
\hline group & Ref. & $\begin{array}{c}p_{\mathrm{K}} \\
{[\mathrm{GeV} / c]}\end{array}$ & method & $\begin{array}{l}\text { measured } \\
\text { part of } f^{(-)}\end{array}$ & $\begin{array}{l}\chi^{2} / \text { dof } \\
\text { in fit }\end{array}$ \\
\hline Angelopoulos et al. & {$[3]$} & $0.25-0.75$ & $\mathrm{~K}^{0}\left(\overline{\mathrm{K}}^{0}\right) \rightarrow \pi^{+} \pi^{-}$ & $\operatorname{Re}, \operatorname{Im}^{a}$ & $13.1 / 10$ \\
\hline Christenson et al. & {$[4]$} & 1.1 & $\mathrm{~K}_{\mathrm{S}} \rightarrow \pi^{+} \pi^{-}$ & $\bmod$ & $0.1 / 1$ \\
\hline Böhm et al. & {$[5]$} & 2.7 & $\mathrm{~K}_{\mathrm{S}} \rightarrow \pi^{+} \pi^{-}$ & $\bmod$ & $1.7 / 1$ \\
\hline \multirow[t]{3}{*}{ Bott-Bodenhausen et al. } & {$[6]$} & 4.5 & $\mathrm{~K}_{\mathrm{L}, \mathrm{S}} \rightarrow \pi \mu \nu$ & $\arg$ & $1.2 / 1$ \\
\hline & [7] & & $\mathrm{K}_{\mathrm{L}, \mathrm{S}} \rightarrow \pi^{+} \pi^{-}$ & $\bmod$ & $0.0 / 1$ \\
\hline & & & & $\arg$ & $0.8 / 1$ \\
\hline \multirow[t]{3}{*}{ Carithers et al. } & [8] & $4-10$ & $\mathrm{~K}_{\mathrm{L}, \mathrm{S}} \rightarrow \pi \ell \nu$ & $\arg$ & $13.0 / 13$ \\
\hline & [9] & & $\mathrm{K}_{\mathrm{L}, \mathrm{S}} \rightarrow \pi^{+} \pi^{-}$ & $\arg ^{b}$ & $7.9 / 6$ \\
\hline & {$[10]$} & & & $\bmod$ & $6.4 / 6$ \\
\hline \multirow[t]{2}{*}{ Hladky et al. } & {$[11]$} & $10-30$ & $\mathrm{~K}_{\mathrm{L}, \mathrm{S}} \rightarrow \pi^{+} \pi^{-}$ & $\bmod$ & $1.2 / 5$ \\
\hline & & & & $\arg$ & $0.0 / 1$ \\
\hline \multirow[t]{2}{*}{ Albrecht et al. } & {$[12]$} & $16-40$ & $\mathrm{~K}_{\mathrm{L}, \mathrm{S}} \rightarrow \pi^{+} \pi^{-}$ & $\bmod$ & $1.3 / 6$ \\
\hline & & & & $\arg$ & $0.4 / 3$ \\
\hline \multirow[t]{2}{*}{ Roehrig et al. } & [13] & $30-130$ & $\mathrm{~K}_{\mathrm{L}, \mathrm{S}} \rightarrow \pi^{+} \pi^{-}$ & $\bmod$ & $21.6 / 10$ \\
\hline & & & & $\arg$ & $9.0 / 10$ \\
\hline Schwingenheuer et al. & {$[14]$} & $20-160$ & $\mathrm{~K}_{\mathrm{L}, \mathrm{S}} \rightarrow \pi^{+} \pi^{-}$ & $\bmod , \arg { }^{c}$ & $0.3 / 1$ \\
\hline
\end{tabular}

Table 2: Carbon $\mathrm{K}^{ \pm}$experiments.

\begin{tabular}{|c|c|c|c|c|c|}
\hline group & Ref. & $\begin{array}{c}p_{\mathrm{K}} \\
{[\mathrm{GeV} / c]}\end{array}$ & $\begin{array}{l}\text { measured } \\
\text { quantities }\end{array}$ & $\begin{array}{l}\text { derived } \\
\text { part of } f^{(-)}\end{array}$ & $\begin{array}{l}\chi^{2} / \text { dof } \\
\text { in fit }\end{array}$ \\
\hline Bugg et al. & {$[15]$} & $0.6-2.6$ & $\sigma_{\mathrm{T}}\left(\mathrm{K}^{ \pm}\right)$ & $\mathrm{Im}$ & $17.5 / 9$ \\
\hline Abrams et al. & [16] & $1.0-3.3$ & $\sigma_{\mathrm{a}}\left(\mathrm{K}^{ \pm}\right)$ & $\mathrm{Im}$ & $37.8 / 41$ \\
\hline \multirow[t]{2}{*}{ Gobbi et al. } & {$[17]$} & $1.68-2.26$ & $\sigma_{\mathrm{T}}\left(\mathrm{K}^{ \pm}\right)$ & $\mathrm{Im}$ & (discarded) \\
\hline & & & $\mathrm{d} \sigma / \mathrm{d} q^{2}\left(\mathrm{~K}^{ \pm}\right)$ & $\mathrm{Re}$ & (discarded) \\
\hline Afonasyev et al. & {$[18]$} & 1.8 & $\sigma_{\mathrm{T}}\left(\mathrm{K}^{ \pm}\right)$ & $\mathrm{Im}$ & $0.8 / 1$ \\
\hline Backenstoss et al. / & [19] & 0 & $a_{0}\left(\mathrm{~K}^{-}\right)$ & $\mathrm{Im}$ & (fixed) \\
\hline Seki & [20] & & & & \\
\hline
\end{tabular}




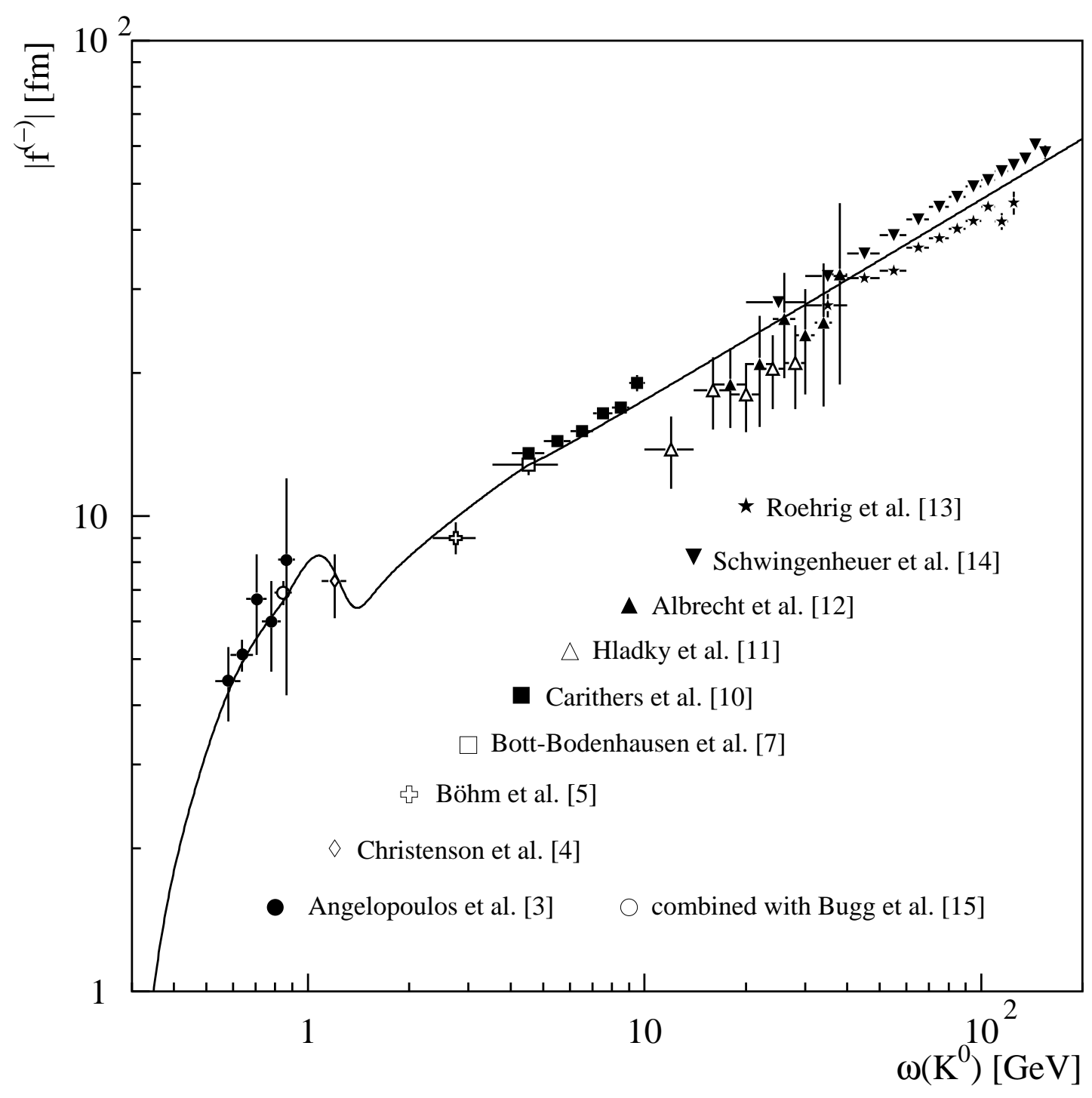

Figure 1: Modulus of the regeneration amplitude $f^{(-)}$: experimental results in comparison with our fit (solid line). The data points of Schwingenheuer et al. are taken from Ref. [27] and are shown just for visualization (only their published value for $\alpha$ enters the fit). Note the doublelogarithmic scale. 


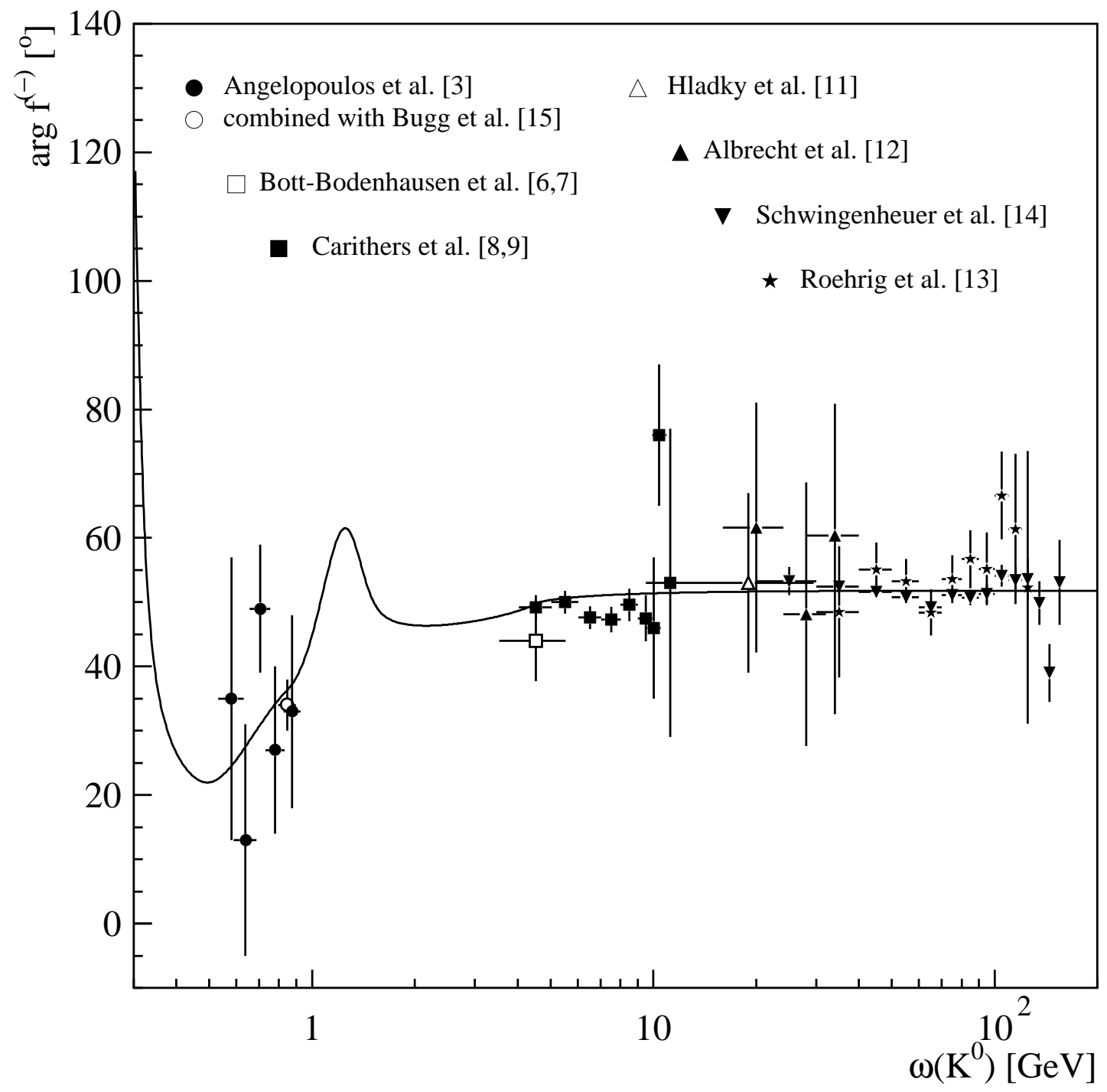

Figure 2: Phase of the regeneration amplitude $f^{(-)}$: experimental results in comparison with our fit (solid line). The data points of Schwingenheuer et al. are taken from Ref. [27] and are shown just for visualization (only their published value for $\alpha$ enters the fit). The data of Refs. [6] and [7] as well as those of Refs. [8] and [9] have been averaged for clarity. Note the logarithmic scale. 




Figure 3: Imaginary part of the regeneration amplitude $f^{(-)}$: experimental results in comparison with our fit (solid line) and the optical model calculation of Ref. [26] (dashed line). The dotted line represents the sub-threshold parameterization employed in Ref. [24]. The data of Ref. [17] are discarded in the fit. Note the linear scale. 


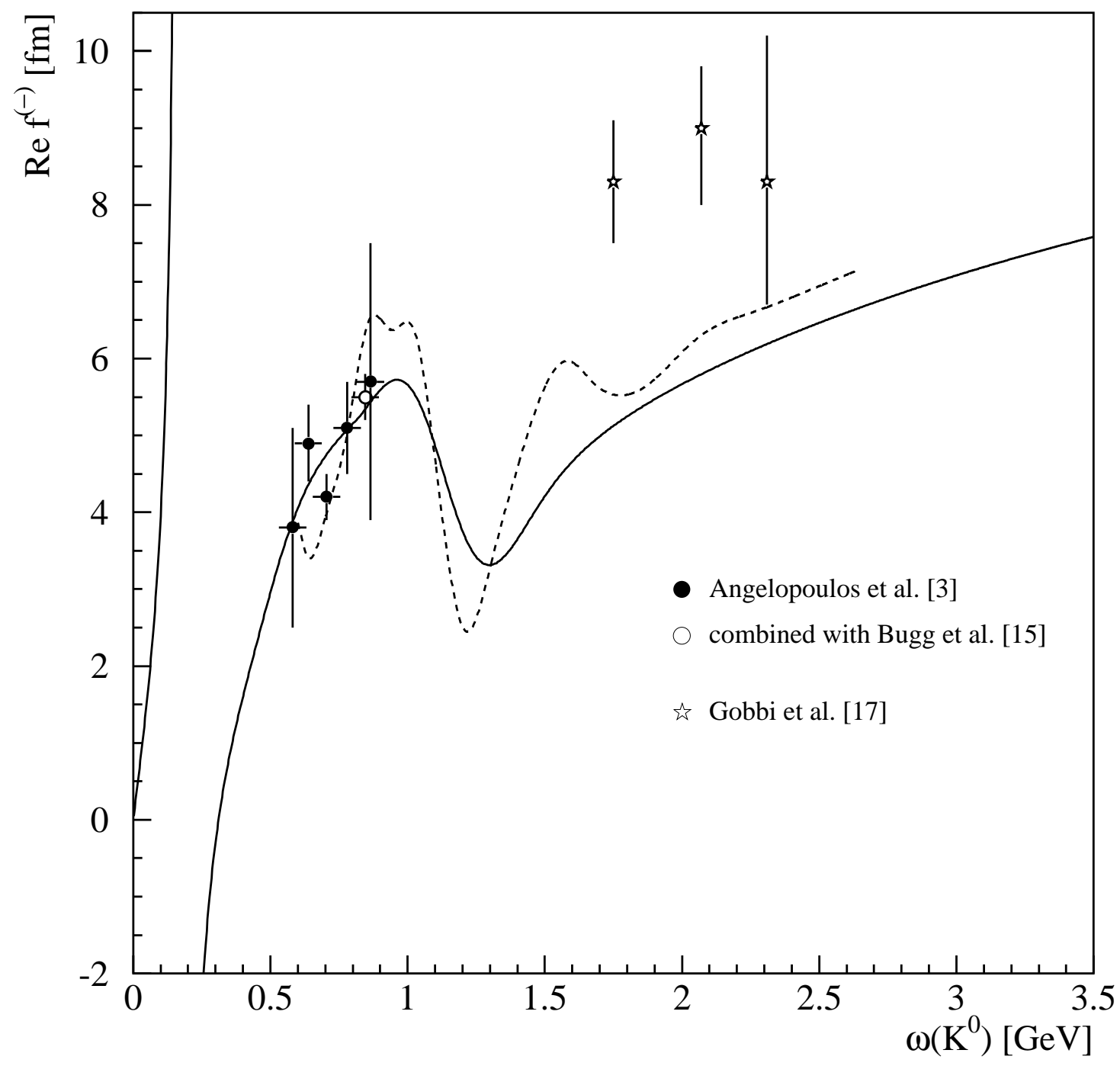

Figure 4: Real part of the regeneration amplitude $f^{(-)}$: experimental results in comparison with our fit including the pole at $\omega_{\mathrm{p}}=172.3 \mathrm{MeV}$ (solid line) and the optical model calculation of Ref. [26] (dashed line). The data of Ref. [17] are discarded in the fit. Note the linear scale. 
$99 / 03 / 07 \quad 18.38$

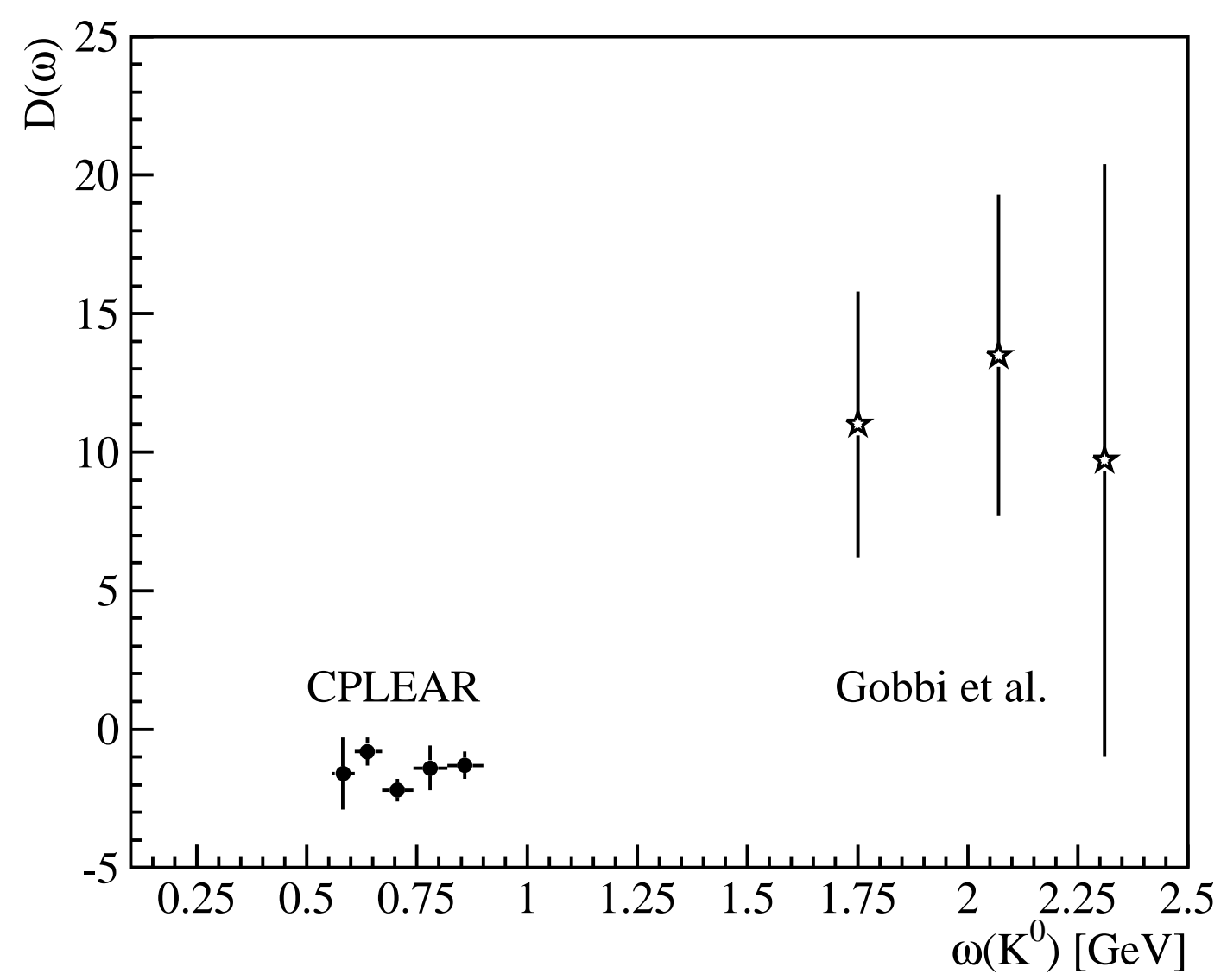

Figure 5: The function $D(\omega)$ as derived from our data (full circles) and from the data of Ref. [17] (see text). 
a)

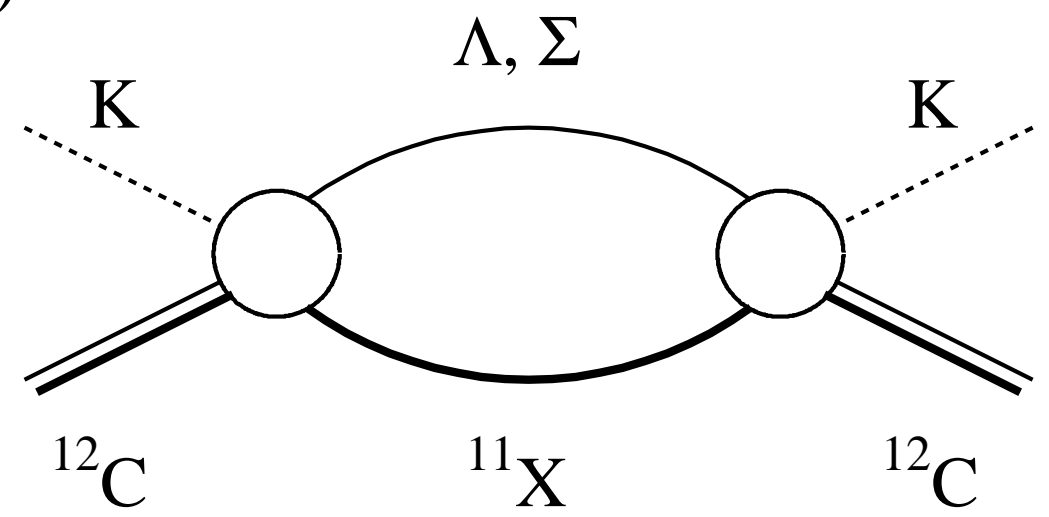

b)



Figure 6: $\mathrm{K}^{12} \mathrm{C}$ scattering diagrams for hyperon (a) and pion (b) exchange. ${ }^{11} \mathrm{X}\left({ }^{12} \mathrm{X}_{\Lambda}\right)$ denotes a spectator system of 11 nucleons (and a $\Lambda$ hyperon). 